\title{
A speeded coding task using a computer-based mouse response
}

\author{
JASON MCPHERSON and NICHOLAS R. BURNS \\ University of Adelaide, Adelaide, Australia
}

\begin{abstract}
This study assessed whether a speeded coding task that used a computer-based mouse response (CBMR) format was a measure of general processing speed (Gs). By analyzing the task within a network of tasks representing both traditional Gs tests and reaction time tasks, it was shown that a CBMR test can be used to measure the same construct as traditional paper-and-pencil (PP) tests and that this response format does not introduce variance associated with psychomotor performance. Differences between PP and CBMR formats were observed, and it is argued that these may provide information on individual differences in performance not available from traditional coding tests.
\end{abstract}

Research articles concerned primarily with coding tasks (or substitution tasks, as they are also known) usually begin with some reference to the long-standing and widespread use of such tasks within psychology. These statements appear to be justified. Coding tasks have been used by researchers investigating the cognitive abilities underlying psychometric intelligence (Ackerman, Beier, \& Boyle, 2002; Burns \& Nettelbeck, 2003; Gignac \& Vernon, 2003), aging processes (MacDonald, Hultsch, Strauss, \& Dixon, 2003; Salthouse, Letz, \& Hooisma, 1994), psychopharmacological effects (Hallam, Olver, McGrath, \& Norman, 2003; Mattila, Aranko, Mattila, \& Paakari, 1994), health-related cognitive impairment (Deary, Sommerfield, McAulay, \& Frier, 2003), and attention (Bate, Mathias, \& Crawford, 2001; Pogge, Stokes, \& Harvey, 1994). These tasks, the most well known being the digit symbol task from the Wechsler intelligence scales, have their roots in the coding subtest of the Army Beta battery developed around 1917, which in turn was based on an earlier test devised by Otis (Matarazzo, 1972).

The digit symbol task is a paper-and-pencil (PP) coding task requiring the association of symbols with digits by reference to a code table. Performance on this task is measured as the number of correctly associated symbols placed beneath a series of digits in a set amount of time - typically, 90-120 sec. The digit symbol task consists of a series of homogenous items of trivial difficulty administered under strict time constraints. These features are common to tests loading on general processing speed (Gs) within contemporary factor-analytic theories of intelligence. Such tests are commonly referred to as speed tests.

The research reported here was supported by Australian Research Council Discovery Grant DP0211113, held by the second author. Correspondence concerning this article should be addressed to J. McPherson, Department of Psychology, University of Adelaide, Adelaide, SA 5005, Australia (e-mail: jlmcpher@psychology.adelaide.edu.au).
There has been considerable research investigating the relationship between PP and computer-based (CB) presentations of speed tests (see Mead \& Drasgow, 1993, for a review). The most notable finding is that the cross-modal (PP with $\mathrm{CB}$ ) correlations for speed tests tend to be lower than those for power tests (in which items of varying difficulty are administered with minimal time constraints). Mead and Drasgow estimated a cross-modal correlation of . 72 for speed tests and .97 for timed power tests. The reason for the lower correlation for speed tests, according to the authors, is that perceptual and motor processes contribute more variance in speed tests than in power tests and that even minor alterations to the presentation and response formats will affect these processes. This is supported by research demonstrating the sensitivity of speeded tests to even slight modifications to response formats (Wegner \& Ree, 1985).

In the past, researchers who have developed computerized coding tasks have opted for a keyboard response format (Baker, Letz, \& Fidler, 1985; Laux \& Lane, 1985; Mattila et al., 1994; Salthouse et al., 1994). These CB tests have achieved a number of stated goals, such as allowing the automation of scoring, allowing dynamic manipulation of task components, and being sensitive to the effects of drugs (Mattila et al., 1994) and aging (Salthouse et al., 1994). However, it can be argued that using a keyboard response format is discrepant with the way that most people interact with modern computers. Indeed, research investigating eye gaze movements during a CB coding task, found that approximately one third of the response time was associated with looking at the keyboard (Stephens, 2001). It would thus seem that although some people may find the keyboard a comfortable way to respond - those who can touch type, for instance - many others may find the keyboard an awkward way to respond.

It is our view that the computer mouse provides a more natural and ecologically valid response format than does the keyboard: first, because it is the most common device for interacting with a computer used by the general public, 
and second, because it allows a person to keep his or her eyes fixed on the screen, regardless of level of computer experience. Previously, many experimental tasks were programmed as, for example, DOS-based applications and were, therefore, most amenable to keyboard response formats. However, the general public has long ago abandoned such operating systems in favor of those with a graphic user interface. Just as handwriting was the natural response format for PP tests, we believe that the mouse is the natural response format for $\mathrm{CB}$ tests.

The general aim of the present study was to develop a speeded coding test utilizing a $\mathrm{CB}$ mouse response (CBMR) format. Despite the evidence for mode of presentation effects in $\mathrm{CB}$ tests, we believe that the important question is whether a $\mathrm{CB}$ coding test can provide a measure of the underlying psychological construct of interest (i.e., Gs). We assume that any test using a CBMR format will have unique variance associated with mouse use, just as PP tests have unique variance associated with the graphomotor elements of such tests (Laux \& Lane, 1985). However, we do not view this source of variance unfavorably, because, with the increasing use of computers, the inclusion of this variance may potentially increase the ecological validity of Gs tests for some purposes (e.g., personnel selection).

Given the identification of the digit symbol task as a strong indicator of Gs within contemporary theories of intelligence (McGrew \& Flanagan, 1998), it is envisaged that a CBMR coding task should also measure this latent construct. However, even if a CBMR test shares considerable variance with traditional Gs tests, the nature of a mouse response format may potentially introduce variance due to psychomotor abilities, such as those commonly measured by reaction time tasks. For this reason, the present study assessed the relationship of a CBMR coding task with both traditional tests of Gs and with paradigmatic reaction time tasks using both exploratory and confirmatory factor-analytic procedures, as well as simple correlational analysis. In addition, the relationships between response formats in PP and CB coding tasks were explored, using subtask presentations for each modality.

\section{METHOD}

\section{Participants}

There were $N=49$ participants who were predominantly Level 1 psychology students from the University of Adelaide, who received course credit for their participation. The mean age was 21.2 years $(S D=4.32) ; 37$ of them were female $(M=21.4$ years, $S D=4.5)$, and 12 were male $(M=20.7$ years, $S D=3.8)$.

\section{Materials}

PP tests are denoted by the suffix PP. CB tests using a mouse response format are denoted CBMR.

\section{Tests of Gs}

Digit Symbol (DS-PP). This test from the Wechsler Adult Intelligence Scales (WAIS-III) required the filling in of blank cells according to a key provided at the top of each test sheet. The standard 2-min time limit was used.

Visual Matching (VM-PP). This test, taken from the Woodcock-Johnson Psycho-Educational Battery-Revised (Woodcock \& Johnson, 1989), required searching for and circling matching pairs of numbers. The standard 3-min time limit was used.

\section{Customized Tests}

Symbol Symbol (SS-PP). This PP test was designed to be as similar as possible to the DS-PP test, but with the coding element of the task removed. The test was structured in the same way as the DS-PP, except that symbols were placed in the upper squares and the participants were simply required to copy these as quickly as possible. A time limit of 1 min was used to minimize fatigue, because piloting indicated heavier physical demands for this test than

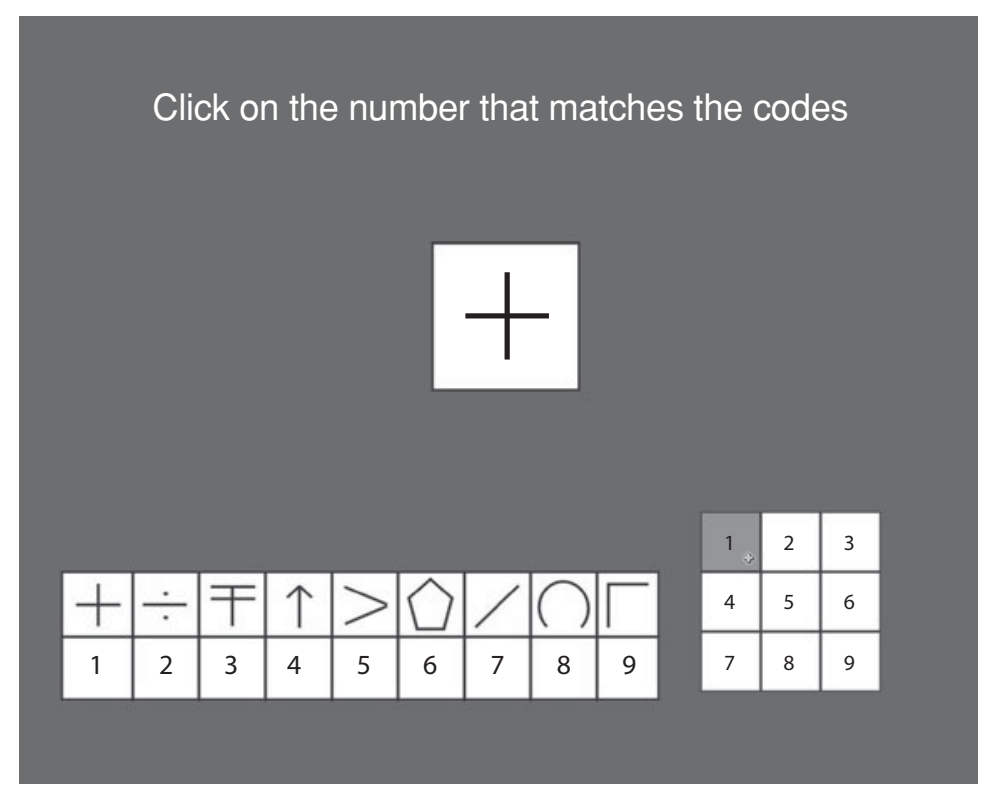

Figure 1. Screen shot from the Symbol Digit (SD-CBMR) test. 
for the other PP tests. The series of items matched the correct series of responses required in the DS-PP.

Symbol Digit (SD-CBMR). The layout for this computerized test is shown in Figure 1. Each item consisted of a symbol presented in the center of the screen, situated within a white box with a black border $(5.4 \times 5.2 \mathrm{~cm})$. The participants responded by left clicking the mouse on a $3 \times 3$ numerical grid $(6.8 \times 6.2 \mathrm{~cm})$ in the bottom right-hand corner of the screen. To make the use of this number grid easier, each number's background turned red when the cursor was placed over it, and the cursor was restricted such that it could not be moved outside of the grid area. The correct response was determined according to the code table $(22.1 \times 4.7 \mathrm{~cm})$ at the bottom left-hand side of the screen. When a correct response was made, a "laser" style sound was heard, and the next item appeared in the center of the screen. When an incorrect response was made, the computer's default beep sound was heard, and the item remained until the correct response was made. The background color of the screen was blue. Seven practice items were provided before the test began. The participants were instructed to complete as many items as they could in $2 \mathrm{~min}$. The sequence of responses required was the same as that for the DS-PP.

Digit Digit (DD-CBMR). This test was designed to be as similar as possible to the SD-CBMR, above, but with the coding element removed. The layout of this computerized test is shown in Figure 2. Each item consisted of a single digit (between 1 and 9) in Arial Black font situated within a white box with a black border $(5.4$ $\times 5.2 \mathrm{~cm}$ ). The participants responded by left clicking the mouse on the corresponding number, using the same numerical grid as that for SD-CBMR. All other aspects of the response format also were the same as those for the SD-CBMR. Seven practice items were provided before the test began. The participants were instructed to complete as many items as they could in $2 \mathrm{~min}$. The sequence of items was the same as that for the DS-PP.

Both computerized tests were run on a Pentium 4 class computer (2.4 MHz) with a standard Microsoft mouse, which was brand new at the start of the experiment. Display was via a 19-in. monitor with a vertical refresh rate of $75 \mathrm{~Hz}$ and a screen resolution of $1,280 \times$ 1,024 pixels.

\section{Reaction Time Tests}

Simple Reaction Time (SRT). This test was based on the task described by Jensen (1982). The display and response panel was as described by Jensen and Munro (1979) but scaled down so that the surface of the panel measured $23 \mathrm{~cm}$ wide and $15 \mathrm{~cm}$ deep; the panel was angled at $30^{\circ}$ to the surface of the table. The participants were required to hold down a single button and then release it as quickly as possible after the light above it came on. There were 10 practice trials and 60 experimental trials. The single dependent measure taken from this task was the median time to respond after the onset of the stimulus.

Odd-Man-Out Reaction Time. This test was based on the task described by Frearson (Frearson, Barrett, \& Eysenck, 1988; Frearson \& Eysenck, 1986) and used the same apparatus as that for the SRT, above. For each stimulus, three of a possible eight lights were lit. One light was the odd man out (OMO) that is, two lights were closer together, with one farther away from the pair. The participant responded by pressing the button below the light. There were 20 practice trials and 60 experimental trials. The task returns two dependent variables: median decision time (OMO-DT), measured from the onset of the stimulus array until the participant lifted the finger from the home button, and median movement time (OMOMT), measured from release of the home button until the target button was pressed.

\section{Procedure}

The participants were tested individually in a quiet room and were asked to concentrate as best they could for each individual test. It was further emphasized that it was important for them to do their best on each test so that an accurate comparison could be made across their performances on all tasks. The tests were administered to all the participants in the following order: SS-PP, VM-PP,

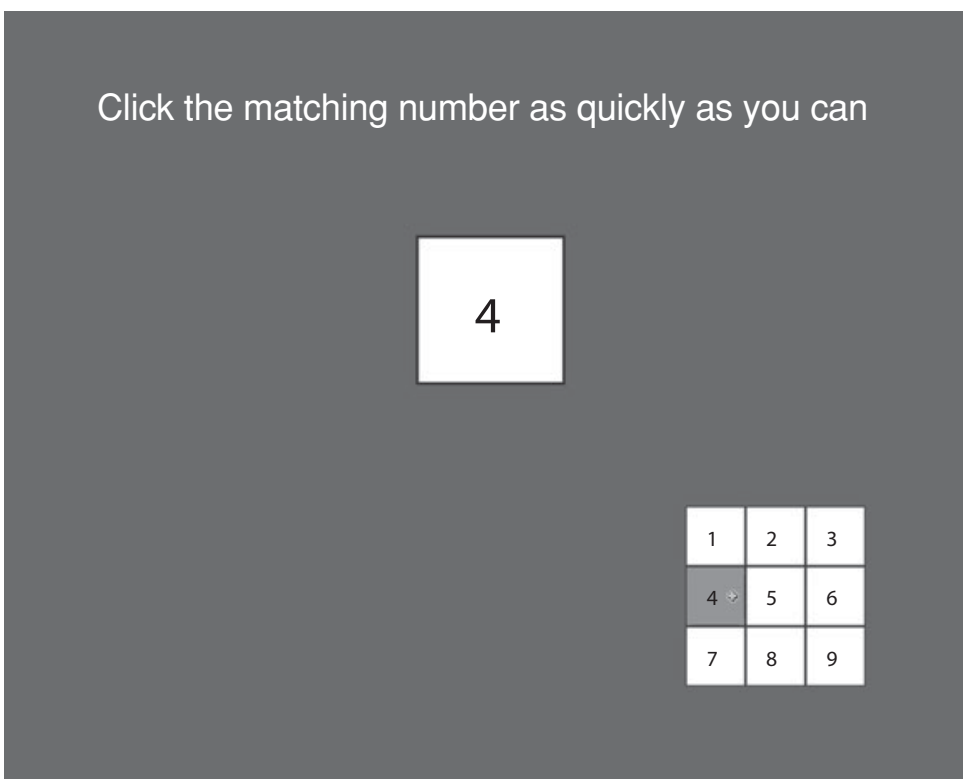

Figure 2. Screen shot from the Digit Digit (DD-CBMR) test. 
Table 1

Descriptive Statistics for Reaction Time, Paper-and-Pencil (PP), and Computer-Based Mouse Response (CBMR) Tests (Number of Correct Items)

\begin{tabular}{lrrcr}
\hline \multicolumn{1}{c}{ Variable } & \multicolumn{1}{c}{$M$} & \multicolumn{1}{c}{$S D$} & Minimum & Maximum \\
\hline Digit Symbol (DS-PP) & 89.8 & 12.5 & 67 & 124 \\
Visual Matching (VM-PP) & 56.0 & 6.1 & 42 & 67 \\
Symbol Symbol (SS-PP) & 95.5 & 13.2 & 68 & 133 \\
Symbol Digit (SD-CBMR) & 86.4 & 15.9 & 61 & 128 \\
Digit Digit (DD-CBMR) & 55.9 & 6.1 & 42 & 67 \\
Simple Reaction Time (SRT) & 218.5 & 25.2 & 170 & 299 \\
$\begin{array}{l}\text { Odd-Man-Out Decision Time } \\
\quad \text { (OMO-DT) }\end{array}$ & 531.6 & 116.3 & 351 & 929 \\
$\begin{array}{l}\text { Odd-Man-Out Movement Time } \\
\quad \text { (OMO-MT) }\end{array}$ & 206.8 & 42.1 & 130 & 320 \\
\hline
\end{tabular}

DS-PP, DD-CBMR, SD-CBMR, SRT, and OMO Reaction Time (OMO-DT and OMO-MT).

\section{RESULTS}

\section{Descriptive Statistics}

Descriptive statistics for all tests are presented in Table 1. There were no obvious ceiling or floor effects evident in any of the test measures. Considering only the coding tasks, the mean DS-PP score was slightly higher than the mean SD-CBMR score $[t(48)=1.96, p=.056$; $d=0.57]$. However, SD-CBMR scores had a larger range than did DS-PP scores, the SD-CBMR test having a lower minimum, a higher maximum, and a correspondingly higher standard deviation. Inspection of the distributions for each test suggested that SD-CBMR test scores were more positively skewed than the DS-PP scores, with skewness coefficients of .63 and .27 , respectively.

\section{Correlations}

Correlations between test scores and age indicated age-related effects that were not consistent across tests.
For example, the correlations with age for OMO-DT and SD-CBMR were .61 and .06, respectively. For this reason, test score variables were age residualized for subsequent analyses. The correlations between all the tests are presented in Table 2.

The two Gs marker tests, the VM-PP and DS-PP, were correlated at .55, and the largest correlation in the matrix is between the DS-PP and the SD-CBMR. The correlations between the two Gs marker tests and the new SD-CBMR test indicate substantial common variance between these three tests. The reaction time tasks appear to share common variance, indicated by the pattern of small-to-moderate significant intercorrelations of these tasks and their near-zero correlations with the PP and CBMR tests.

\section{Exploratory Factor Analysis}

The validity of SD-CBMR as a measure of Gs was assessed using exploratory factor analysis (EFA). This was done prior to any confirmatory factor analysis (CFA), to allow any inherent structure in the data to be assessed independently of theoretical expectations. The SD-CBMR test, together with the three reaction time tasks and the two Gs marker tests, was entered into an initial principal components analysis. There were two components with eigenvalues larger than one (2.28 and 1.57), accounting for $38.0 \%$ and $26.2 \%$ of the variance, respectively. Inspection of the scree plot also indicated two dominant components. With the eigenvalue and scree criteria as an initial guide, and in an attempt to avoid an underestimation of underlying factors (Fabrigar, Wegener, MacCallum, \& Strahan, 1999), it was decided to begin further analysis with the extraction of three factors.

Because we were interested primarily in the latent structure of the data and given that skew and kurtosis for all of the variables were below one, maximum likelihood extraction was used (Fabrigar et al., 1999). An attempt to extract three factors resulted in a Heywood case (estimated communality for a variable larger than one) and

Table 2

Intercorrelations for Reaction Time, Paper-and-Pencil (PP), and Computer-Based Mouse Response (CBMR) Tests

\begin{tabular}{|c|c|c|c|c|c|c|c|c|c|c|}
\hline Variable & 1 & 2 & 3 & 4 & 5 & 6 & 7 & 8 & 9 & 10 \\
\hline 1. Digit Symbol (DS-PP) & 1 & & & & & & & & & \\
\hline 2. Visual Matching (VM-PP) & $.55^{* *}$ & 1 & & & & & & & & \\
\hline 3. Symbol Symbol (SS-PP) & $.45^{* *}$ & $.42^{* *}$ & 1 & & & & & & & \\
\hline 4. Symbol Digit (SD-CBMR) & $.67^{* *}$ & $.50^{* *}$ & .12 & 1 & & & & & & \\
\hline 5. Digit Digit (DD-CBMR) & $.51^{* *}$ & $.35^{*}$ & .26 & $.56^{* *}$ & 1 & & & & & \\
\hline 6. Simple Reaction Time (SRT) & .01 & .01 & -.06 & -.01 & -.05 & 1 & & & & \\
\hline $\begin{array}{l}\text { 7. Odd-Man-Out Decision Time } \\
\text { (OMO-DT) } \\
\text { 8. Odd-Man-Out Movement Time }\end{array}$ & -.13 & -.13 & .08 & -.16 & -.14 & $.30^{*}$ & 1 & & & \\
\hline $\begin{array}{l}\text { (OMO-MT) } \\
\text { 9. Symbol Digit Error Rate }\end{array}$ & -.06 & -.13 & -.18 & -.20 & -.17 & $.42^{* *}$ & .28 & 1 & & \\
\hline $\begin{array}{l}\text { (SDER-CBMR) } \\
\text { 10. Digit Digit Error Rate }\end{array}$ & -.17 & -.09 & -.05 & -.12 & .07 & -.12 & -.07 & .06 & 1 & \\
\hline (DDER-CBMR) & $.36^{*}$ & .25 & .21 & $.37^{* *}$ & $.33^{*}$ & $-.36^{*}$ & -.26 & $-.34^{*}$ & $.48^{* *}$ & 1 \\
\hline
\end{tabular}


Table 3

Factor Loadings of Test Variables for Maximum Likelihood Extraction With Varimax Rotation

\begin{tabular}{|c|c|c|}
\hline Variable & Factor 1 & Factor 2 \\
\hline Digit Symbol (DS-PP) & .857 & -.012 \\
\hline Visual Matching (VM-PP) & .633 & -.077 \\
\hline Symbol Digit (SD-CBMR) & .783 & -.138 \\
\hline Simple Reaction Time (SRT) & .055 & .629 \\
\hline Odd-Man-Out Decision Time (OMO-DT) & -.141 & .439 \\
\hline Odd-Man-Out Movement Time (OMO-MT) & -.088 & .670 \\
\hline
\end{tabular}

Note-Bold numbers indicate salient loadings.

a third factor almost identical to the first factor. Extracting two factors resulted in a nonsignificant chi-square $\left[\chi^{2}(4)=1.31, p=.860\right]$, indicating an acceptable fit. Direct Oblimin rotation resulted in two clearly defined factors $(r=-.19)$, each saliently loaded by three tests; largely identical results were obtained with a Varimax rotation. A one-factor solution indicated small loadings for the reaction time tests, and the chi-square $\left[\chi^{2}(9)=15.92\right.$, $p=.068]$ suggested a relatively poorer fit. The two-factor Varimax solution was thus preferred on grounds of fit, simplicity, and interpretability. The pattern of factor loadings for the final solution is presented in Table 3 .

Factor 1 is saliently loaded by the two Gs marker tests and the SD-CBMR, with very small loadings from the three reaction time tests. This factor is thus interpreted as Gs. Factor 2 is saliently loaded by the three reaction time variables, with very small loadings from the other tests. This factor is interpreted as a reaction speed factor. This pattern of factor loadings supports the validity of the SD-CBMR test as a Gs measure.

\section{Confirmatory Factor Analysis}

CFA was undertaken to assess the fit of a measurement model based on the EFA results. First, a two-factor model was specified in which the three reaction time measures were allowed to load on one latent variable and the two Gs markers and the SD-CBMR test were allowed to load on the other. The latent variables were specified as independent. The fit of this model to the data was very good $\left[\chi^{2}(9)=4.26, p=.894 ;\right.$ GFI $=.972$, RMSEA $<$ $.001]$. To assess the potential relationship of reaction time speed with SD-CBMR, the initial two-factor model was modified by allowing the SD-CBMR to load on both latent variables. If the SD-CBMR shared any substantial variation with the reaction time tests, we would expect an improvement in model fit. This respecified model also fit the data well $\left[\chi^{2}(8)=3.27, p=.917\right.$; GFI $=.979$, RMSEA $<.001]$, but a nested model test indicated that the chi-square difference was nonsignificant $\left[\Delta \chi^{2}(1)=\right.$ $.99, p=.317]$. The estimated path loading between the latent reaction time variable and the SD-CBMR was -.14 . These results further support the validity of the SD-CBMR as a measure of Gs that is unconfounded with reaction time.

\section{Subtask Analyses}

The SS-PP and DD-CBMR tests served as subtasks of the DS-PP and SD-CBMR tests, respectively, in which the same presentation and response formats were used, but with no coding of stimuli required. It can be seen in Table 2 that these two subtasks are moderately correlated $(r=.26)$ but that they are more strongly related to their corresponding full-task equivalents. The SS-PP test does not appear to have a substantial relationship with the computerized coding task, SD-CBMR $(r=.12)$, whereas the DD-CBMR seems to share considerable variance with the DS-PP $(r=.51)$ and, to a lesser extent, the VM-PP $(r=.35)$.

To compare the mean scores for each subtask we multiplied the SS-PP scores by two, to adjust for the 1-min time limit. The adjusted mean SS-PP score (190.1, SD = 26.4) was clearly higher than the mean DD-CBMR score $[t(48)=13.1, p<.001 ; d=3.8]$.

\section{Analysis of Errors}

One further observed difference between the test modalities was the error rate. In all of the PP tests, the number of errors made were so few as to make any analysis of individual differences meaningless. However, using the mouse response formats, the participants varied more considerably on the numbers of errors made in the DD$\operatorname{CBMR}(M=4.0, S D=4.8)$ and DS-CBMR $(M=5.3$, $S D=5.3)$. These error scores were converted to error rates in order to control for speed-accuracy trade-off and then were age residualized to make these measures consistent with the other test measures. Error rates for each of the CBMR tests were correlated at .48, indicating a moderate level of shared variance, but a susbtantial amount of unique variance as well. This is further evident in the differential correlations with the other tests. Error rates for the DD-CBMR had larger correlations with other test measures than error rates for the SD-CBMR had with the same tests.

\section{DISCUSSION}

This study assessed the validity of a coding task that used a CBMR format as a measure of Gs. By analyzing the task within a small network of tasks representing both traditional Gs tests and reaction time tasks, it was shown that a CBMR test can be used to measure the same construct as traditional PP tests and that this response format does not introduce variance associated with psychomotor performance. These conclusions are supported by correlational and exploratory and confirmatory factor-analytic results.

The test developed for the present study, the SD-CBMR, had correlations with the two PP Gs marker tests that were similar to those that these two tests had with each other. Furthermore, these correlations were possibly attenuated in the largely university-based sample. Thus, the correlation between the SD-CBMR and the traditional Gs measures in a more representative sample could potentially be 
larger than was estimated in the present study. Despite this possibility, the intercorrelations between tasks were large enough for a distinct pattern to emerge in EFA.

The EFA solution suggested two common factors underlying the six speed tests. We interpreted the first factor as Gs and the second as a reaction time factor. The SD-CBMR was clearly loaded on Gs and not on the reaction time factor, and this finding was supported in the subsequent CFA. These results illustrate the advantages of using factor-analytic techniques and support Mead and Drasgow's (1993) appeal for the use of such methods. However, despite the similarities between the PP and the CBMR tests, it should be noted that a number of differences were observed in the present study.

Test scores from the DS-CBMR were slightly lower than those for the DS-PP, which indicates that the CBMR coding task may be more difficult in some way. In parallel with this result, the PP subtask, the SS-PP, had an even higher mean relative to the CBMR subtask, the DDCBMR. This suggests that for most people, the CBMR format was slower than the traditional PP response format. However, the larger range and skew of scores for the SDCBMR test suggest that this was not the case for all people. Interpretation of these differences is made difficult by the fact that PP and CBMR tests were differentiated by more than the use of a mouse or a pencil. First, each test modality required differential perceptual scanning. In the traditional DS-PP, the scanning distance from item to code table and back alters as one progresses through the test. Second, the traditional test also permits people to use their previous responses as an additional and potentially more convenient code table proxy. Both of these factors, in addition to the use of the mouse, could have contributed to differences in test means. This is further supported by the intercorrelations of the two subtasks with other tests.

The subtasks for each modality, the SS-PP and DDCBMR, were not highly correlated with one another, suggesting that they do not tap a common speed construct. However, each test had more substantial relationships with other tests. The SS-PP was moderately but significantly correlated with the DS-PP and the VM-PP, whereas it had a negligible relationship with the SD-CBMR. It thus seems likely that the relationship between the SS-PP and the two PP Gs measures was due largely to the graphomotor and feature-encoding requirements in these tests. In contrast, the DD-CBMR was more substantially correlated with all of the tests that loaded on Gs, particularly the DS-PP and SD-CBMR. This suggests that the DDCBMR required some ability that all the Gs tests shared, especially the coding tasks.

Although the DD-CBMR would not seem to require the type of perceptual scanning that coding tasks do, it does require rapid eye movement between the center of the screen and the response grid and the subsequent movement of the cursor to the correct digit. The most obvious suggestion, then, is that these visual requirements of the DD-CBMR are related to those required in coding tasks in general. In this sense, the subtask was not entirely suc- cessful, in that it did not serve as a satisfactory measure of simple response speed. However, the analysis of errors in the CBMR tasks does provide some additional insight into the differences between PP and CBMR formats.

It was clear that the CBMR format evoked higher error rates than did the traditional PP formats, which are generally found to have negligible error rates. The first conclusion that might be drawn about the error rates is that it is easier to make an error using the CBMR response format. Although the CBMR format evoked slower performances overall, the final action of clicking the mouse is potentially quicker than the drawing of a symbol and may, thus, be more prone to errors associated with speedaccuracy trade-offs. Furthermore, although the error rates in the SD-CBMR and the DD-CBMR were similar, their intercorrelations with other measures suggest that error rates from the DD-CBMR were more substantially related to other speed tests. One possible interpretation of this is that the simpler DD-CBMR is more sensitive to a rapid responding style that may lead to speed-accuracy trade-offs.

However, despite all of this, error rates in the SD-CBMR were minimally correlated with every measure except the error rate from the DD-CBMR. This moderately high correlation of error rates for each CBMR test is reminiscent of so-called carelessness constructs that have been identified in factor-analytic studies of attentional abilities (see Carroll, 1993, for a review). Thus, although the SD-CBMR scores do not appear to confound speed-accuracy tradeoffs with overall speed scores, error rates from the test may potentially provide additional information about a person's response style and attentional abilities.

Although using a CBMR format is unlikely to provide a parallel form of any traditional speed test, the present study suggests that this format can be used in the assessment of speeded ability constructs. However, the differences between traditional PP and CBMR formats should not be overlooked, because they may prove useful in some instances and raise problems in others. In addition, before CBMR formats can be more generally accepted, further research will be necessary to assess the external validity of any tests that utilize such a response format. Moreover, issues on the quality and durability of the computer mouse require consideration. We used a brand new conventional mouse but acknowledge that this type of mouse is prone to become "gummed-up." We believe an optical mouse would overcome this problem. Another, but less accessible, alternative would be to explore the use of a touch screen response method. Presently, though, we remain optimistic that the psychological assessment of speeded constructs can cautiously move with the technologies that now surround us.

\section{REFERENCES}

Ackerman, P. L., Beier, M. E., \& Boyle, M. O. (2002). Individual differences in working memory within a nomological network of cognitive and perceptual speed abilities. Journal of Experimental Psychology: General, 131, 567-589.

BAKER, E. L., LETZ, R., \& FidLER, A. T. (1985). A computer-administered 
neurobehavioral evaluation system for occupational and environmental epidemiology: Rationale, methodology and pilot study results. Journal of Occupational Medicine, 27, 206-212.

Bate, A. J., Mathias, J. L., \& Crawford, J. R. (2001). Performance on the Test of Everyday Attention and standard tests of attention following severe traumatic brain injury. Clinical Neuropsychologist, 15, 405-422.

Burns, N. R., \& NetTELBECK, T. (2003). Inspection time in the structure of cognitive abilities: Where does IT fit? Intelligence, 31, 237-255.

Carroll, J. B. (1993). Human cognitive abilities: A survey of factor analytic studies. New York: Cambridge University Press.

Deary, I. J., Sommerfield, A. J., McAulay, V., \& Frier, B. M. (2003) Moderate hypoglycaemia obliterates working memory in humans with and without insulin treated diabetes. Journal of Neurology, Neurosurgery, \& Psychiatry, 74, 278-279.

Fabrigar, L. R., Wegener, D. T., MacCallum, R. C., \& Strahan, E. J. (1999). Evaluating the use of exploratory factor analysis in psychological research. Psychological Methods, 4, 272-299.

Frearson, W., Barrett, P., \& EysencK, H. J. (1988). Intelligence, reaction-time and the effects of smoking. Personality \& Individual Differences, 9, 497-517.

Frearson, W., \& EysencK, H. J. (1986). Intelligence, reaction-time (RT) and a new odd-man-out RT paradigm. Personality \& Individual Differences, 7, 807-817.

Gignac, G., \& Vernon, P. A. (2003). Digit Symbol Rotation: A more $g$ loaded version of the traditional Digit Symbol subtest. Intelligence, 31, 1-8.

Hallam, K. T., Olver, J. S., McGrath, C., \& Norman, T. R. (2003). Comparative cognitive and psychomotor effects of single doses of Valeriana officianalis and triazolam in healthy volunteers. Human Psychopharmacology, 18, 619-625.

JENSEN, A. R. (1982). Reaction time and psychometric $g$. In H. J. Eysenck (Ed.), A model for intelligence (pp. 93-132). Heidelberg: Springer.

Jensen, A. R., \& Munro, E. (1979). Reaction time, movement time and intelligence. Intelligence, 3, 121-126.

LAUX, L., \& LANE, D. (1985). Information processing components of substitution test performance. Intelligence, 9, 111-136.
MacDonald, S. W. S., Hultsch, D. F., Strauss, E., \& Dixon, R. A. (2003). Age-related slowing of digit symbol substitution revisited: What do longitudinal age changes reflect? Journal of Gerontology, 58B, 187-194.

Matarazzo, J. D. (1972). Wechsler's appraisal of adult intelligence. Baltimore: Williams \& Wilkins.

Mattila, M. J., Aranko, K., Mattila, M. E., \& PaAkari, I. (1994) Effects of psychotropic drugs on digit substitution: Comparison of the computerized symbol-digit substitution and traditional digit-symbol substitution tests. Journal of Psychopharmacology, 8, 81-87.

McGrew, K., \& Flanagan, D. P. (1998). The intelligence test desk reference (ITDR): Gf-Gc cross-battery assessment. Boston: Allyn \& Bacon.

Mead, A. D., \& Drasgow, F. (1993). Equivalence of computerized and paper-and-pencil cognitive ability tests: A meta-analysis. Psychological Bulletin, 114, 449-458.

Pogge, D. L., Stokes, J. M., \& Harvey, P. D. (1994). Empirical evaluation of the factorial structure of attention in adolescent psychiatric patients. Journal of Clinical \& Experimental Neuropsychology, 16, 344-353.

Salthouse, T. A., Letz, R., \& Hooisma, J. (1994). Causes and consequences of age-related slowing in speeded substitution performance. Developmental Neuropsychology, 10, 203-214.

Stephens, R. (2001). Feasibility of the use of eye movement data in the analysis of neurobehavioral test performance. Environmental Research, 85, 53-57.

Wegner, T. G., \& ReE, M. J. (1985). Armed Services Vocation Aptitude Battery: Correcting the speeded subtests for the 1980 youth population. Brooks Air Force Base, TX: Air Force Human Resources Laboratory, Air Force Systems Command.

Woodcock, R. W., \& Johnson, M. B. (1989). Woodcock-Johnson Psycho-Educational Battery-Revised. Allen, TX: DLM Teaching Resources.

(Manuscript received April 19, 2004; revision accepted for publication July 7,2004 .) 